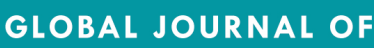 \\ Community Psychology Practice
}

\section{PROMOTING COMMUNITY PRACTICE FOR SOCIAL BENEFIT}

\section{The Community Narration (CN) Approach: Understanding a Group's Identity and Cognitive Constructs through Personal and Community Narratives}

\author{
Bradley D. Olson, PhD \\ Community Psychology, National-Louis University, Chicago, IL, USA \\ Leonard A. Jason, PhD \\ Director for the Center for Community Research, DePaul University, Chicago, IL, USA
}

Key words: Narrative, Participatory, Substance Abuse, Recovery Homes, Oxford House

Brad Olson is Assistant Professor of Psychology at National-Louis University in Chicago and Co-Director of the Community Psychology Doctoral Program. He is a community and social psychologist, and can be reached at bradley.olson@nl.edu.

Leonard Jason, Ph.D. is the Director of the Center for Community Research at DePaul University.

Acknowledgements:

We appreciate the support of Paul Molloy and Leon Venable and the many Oxford House members who have collaborated with our team. The authors are grateful for financial support provided by the National Institute on Alcohol Abuse and Alcoholism (NIAAA grant numbers AA12218 and AA16973), the National Institute on Drug Abuse (NIDA grant numbers DA13231 and DA19935) and the National Center on Minority Health and Health Disparities (grant MD002748).

Recommended citation:

Olson, B.D., \& Jason, L.A. (2011). The community narration (CN) approach: Understanding a group's identity and cognitive constructs through personal and community narratives. Global Journal of Community Psychology Practice, 2(1), 1-7. Retrieved <date>, from http://www.gjcpp.org/. 


\title{
The Community Narration (CN) Approach: Understanding a Group's Identity and Cognitive Constructs through Personal and Community Narratives
}

\author{
Bradley D. Olson ${ }^{1}$, Leonard A. Jason ${ }^{2}$ \\ ${ }^{1}$ National-Louis University, ${ }^{2}$ Center for Community Research, DePaul University
}

\begin{abstract}
Community program evaluations, visioning and assessments must always endeavor to attain useful information in the most sensitive way. Most community-based organizations form, grow and continue on their own without the help of outside experts. Participatory approaches should respect the historical evolution of these groups and understand the positive factors that underlie their organizational beliefs. A group's mission, values and identity should inform any community program evaluation, consulting project, and the design of any research study. Narrative methods have been used with mutual-help groups and many other organizations to good effect (Harré, Bullen, \& Olson, 2006; Rappaport, 2000). Such methods have great potential to avoid hierarchical and unidirectional forms of evaluation, encouraging the group's collective psychology and identity-based constructs to emerge. We developed a participatory, narrative technique called Community Narration $(\mathrm{CN})$, which is described here. The technique utilizes personal stories and community narratives as an entry into the evaluation process or other work involved in understanding an organization. The community's participants were able to use the technique successfully, found it enriching, and the constructs obtained have led to many discussions and memberguided research related to the organization.
\end{abstract}

Community-based techniques such as empowerment evaluation, attempt to reduce hierarchies between the "consultant" and the organization (Fetterman, Kaftarian, \& Wandersman, 1996; Fetterman \& Wandersman, 2005). Whether the goal involves visioning with another organization, creating a needs assessment, or evaluation, there is a need to move toward less rigid methods in order to discover an organization or community's identity, its collective goals, and implicit aspects of its mission. The organizational identity is often built on a collection of personal beliefs and experiences shared by those who make up the community. Personal identities are structured around stories (McAdams, 2009; McAdams \& Olson, 2010). Community narratives, made up of personally stories, are equally the foundation of a group's or community's identity (Harré, Bullen, \& Olson, 2006; Rappaport, 2000).

Rappaport (2000) distinguishes between personal stories and community narratives, associating the story to the individual and the narrative to the community, noting that stories and narratives are intimately tied with one another. Each community has a unique set of narratives that are a source of growth, and a way for a community to creatively find its alternative narratives, which are contrasted with other dominant narratives in society. The contexts and cognitive constructs associated with such alternative and dominant narratives are critical to understanding the relationship or fit between an individual and the larger organization or community (Rappaport, 2000). These understandings can also be instrumental in the recovery path for a group and its members. The formation of these stories, the community narratives, and the constructs that describe them, are most often implicit and remain unidentified. Community psychology can contribute to helping these collective beliefs emerge or become more explicit, a state which may offer great value toward understanding a community and helping that community better understand itself.

From a social as well as a methodological perspective, qualitative approaches have participatory and strengths-based advantages. Telling stories is an enjoyable and enriching experience and community members often report that it feels like a more authentic way of becoming understood from the outside (Harré et al., 2006). Qualitative information also produces rich data that can be looked at as a whole, or coded, and/or combined with quantitative data. Working with groups in a concerted fashion to understand personal and community narratives in concrete terms can help all stakeholders better understand the organization's community and 


\section{Global Journal of Community Psychology Practice}

individual narratives. It is often a good starting point to working with an organization. Unfortunately, qualitative methods in research enterprises can be time and labor intensive (Patton, 2000).

For these reasons, we developed the current Community Narration (CN) approach. The method uses, in part, George Kelly's (1955) cognitive-based clinical-personality model. The goal of Kelly's personal constructs theory is to reduce the personal and community narratives down to shorter and more discussable constructs. The personal construct, the basic unit in George Kelly's (1955) personal construct theory, is represented by dichotomous indicators, most often about other people: "good vs. bad", for example, or "liberal vs. conservative." The contrasts of the bipolar construct add more information than a single term alone. For instance, when discussing food, the bipolar constructs of "hotmild" and "hot-medium" connote a spicy or peppery hot. But "hot-warm" and "hot-cold" connote temperature differences (Maher, 1969). According to Kelly's theory, personal bipolar constructs are the means by which people construe events in the world. In this use, personal construct theory also helps, in the form of manageable dichotomies, bring out the alternative vs. dominant community narratives as described by Rappaport (2000).

A key component of Kelly's (1955) personal construct theory is the "commonality corollary." The corollary suggests that when two or more people employ similar constructions of their experiences, the psychological processes involved are also similar. Commonalities are fundamental to Community Narration and in its goal to derive a group's dichotomous constructs. These bipolar constructs have been used in other, different and more individualistic personality and social role methods of assessment. The procedure of the current Community Narration is described in the Methods section, but, briefly, dichotomies are obtained from group members initially through personal stories, and taken further to uncover central, common community constructs.

Community Narration is useful in helping an organization/community and other stakeholders think about and analyze more implicit aspects of its mission, identity, or what some might call the "culture" of a community or setting (Maton, 2000; Sarason, 1971). Such a method could benefit the group's functioning, its sense of community. Individual members of that setting could better understand their place within that culture, issues of agreement, and the diversity of values of the community. The method is also, therefore, of interest to researchers, evaluators, consultants and other stakeholders.

In the current community the method was on, we hypothesized that constructs would emerge regarding substance use vs. non-use, since this was a group currently in recovery. And "freedom vs. institutionalization", since the model allowed participants to make their own democratic decisions in the mutual-help tradition (alternative or recovery narratives), contrasted to "treatment" options (i.e., more dominant, treatment, or institutional narratives).

\section{Method}

\section{Participants, Organization, and Setting}

While the method works for any organization or community, the group of present interest is based on 12 -step principles. There is a long tradition in the community narrative field to examine mutual-help, and particularly 12-step groups (Humphreys, 2000). Oxford House, a member-run mutual help, residential setting, focuses on recovery from alcohol and drug problems. Neither professionals nor mental health practitioners are directly involved in the operations of the program. It involves communal living. There are over a 1,400 Oxford Houses in the U.S., Canada and Australia. Each house is independent. Yet through a chapter system, houses are connected to the primary organization in the D.C. area. The authors of this paper are part of a university-based research entity. We are organizationally independent of Oxford House, although our relationship with the group, its houses, and individual members organization can often be considered involved and intense (Jason, Davis, Ferrari, \& Bishop, 2001; Olson, Jason, Ferrari, \& Hutcheson, 2005). We do not consider ourselves "consultants" for the organization.

The method was first used and tested with a group of approximately 100 Oxford House residents at the Oxford House World Convention in Washington D.C., a conference where hundreds of Oxford House participants from the U.S., Canada and other nations meet. These conventions include a mix of older and newer residents.

\section{Procedure}

The Community Narrative (CN) program began with members of the research team. We introduced the session as a town-hall style approach to understanding themes of Oxford House community narratives. The following seven phases describe the whole process: 


\section{Global Journal of Community Psychology Practice}

Phase 1-Introduction. The session started with describing the program as a town hall-style meeting, and emphasizing the importance of stories in discovering aspects of individuals and communities. The goal of the session was stated to "...determine common community constructs and to learn, through discussion and storytelling." The goal, in this case of Oxford House residents, was to discover how they construe or perceive their past experiences of having lived in an Oxford House.

Phase 2-Gathering in Subgroups. Attendees sat in chairs in a ballroom style convention hall. We asked the attendees to randomly join groups of 4-6 people, and for those small groups to form circles with their chairs. Subgroup members were asked to introduce themselves.

Phase 3-Sharing Stories. The groups were asked to take roughly five to seven minutes each to share a key personal story that reflected the "high point" (McAdams, 2009), or their most significant experience, in Oxford House. This was to be the single episode that that best defined for them "life in Oxford House". Participants were asked to describe in the story any other characters who had been part of the experience, the participant's own feelings during the event, and why the event was personally important. Sufficient time was given in case some members took longer than seven minutes. The presenters walked around, listening to the discussions, and answering questions if any arose. Eventually group discussions were encouraged to wind down and finish.

Phase 4-Finding Commonalities. The next stage involved finding "commonalities" across the stories within each groups. Group members were asked to collectively identify in the stories a commonality, and to describe that commonality in a single word or two. Reducing the group stories to constructs in this way was somewhat like coding in qualitative analyses, as it involved turning a great deal of verbal text, across participants, into a more manageable whole. Again, the idea behind getting at commonalities, consistent with Kelly's theory, was to obtain fundamental community constructs from the personal constructs. Participants had little trouble identifying these commonalities. When all groups had identified the one or two word terms, they wre asked to announce them out loud to the whole room, and the presenters wrote each term down on the left side of a board, leaving room on the opposite side (on the right side) for where the opposite pole on the bipolar construct would be written. When constructs from different groups appeared were too similar to one another, the constructs were clustered. For instance, one group's commonality term "close friends" and another group's "social connections" were clustered under "support". Other minor clustering occurred. Through this process, the groups generated eighteen primary construct issues.

Phase 5-Choosing Constructs. At this point, presenters directed all attendees to add, collectively across all groups, the contrast terms of the bipolar construct that were, in their minds, the opposite of each of the commonalities the subgroups had developed. These contrasts were written on the right side of the board, forming the second side of the bipolar construct. A "vs." was placed in between the two sets of bipolar terms. As a larger group, the participants had little trouble naming contrast items. There was much certainty, enthusiasm and signs of agreement from multiple participants, seeming to suggest the opposite terms for each main construct were good ones. Eventually, all bipolar constructs were on the sheets standing before the participants.

Phase 6-Voting. All participants were asked to vote as individuals on the constructs they believed best reflected the most significant psychological meaning behind their Oxford House experience. Participants were given time to look over all the constructs, and then told they could vote. It also works to allow participants to vote on their top two constructs, rather than on just one. Each bipolar construct was named by the presenter, and hands were counted. The votes were all collected, and then tallied. Based on the votes received, the bipolar constructs were given a rank from the top to the bottom. This was largely a time-saving measure for the subsequent group discussion phase, where the top constructs would be discussed first.

Phase 7-Group Discussion. Open group discussion was then encouraged around the meaning and relevance of each of the bipolar constructs, starting with the one that received the most votes. The open discussion was important for the whole group to reflect on the rank-ordering, the dichotomies themselves, and their meanings. Discussions around the constructs and their relation to the Oxford House philosophy emerged. Much conversation tended to be around house interactions from first entering the house to personally becoming more stabilized within it. The stories that originally brought about the constructs were re-narrated and new examples were added in.

Ultimately, the top ranked constructs were little more than a focal-point for deeper conversations around the Oxford House experience. Since time permitted, 


\section{Global Journal of Community Psychology Practice}

even those bipolar constructs with the lowest votes ended up getting some discussion, and led to equally interesting ideas, often in how they were similar or different from the top-rated constructs.

\section{Results}

For the purpose of space, the Results will focus on the top four constructs and the interpretations provided by the participants, expanded upon through our own experiences of the group. As seen in Table 1 , the most voted for construct was growth $v s$. stagnation (or regression), which received $21 \%$ of the vote.

According to the participants, growth vs. stagnation (or regression) referred specifically to an increased feeling of social connectedness, a greater personal sense of community, and a new tolerance for others different from oneself. This discussion in fact helped us become interested in how Oxford House may reduce various prejudices in residents, leading to study on the topic (see Olson, Jason, Davidson, \& Ferrari, 2009). One of our hypothesized constructs "sobriety vs. using" came in only eighth place. Therefore we were somewhat surprised for this primary outcome-related topic like abstinence or the ability to stop using drugs to stand so far behind this personal and interpersonal form of "growth" that emerged as the most central Oxford House process.

The second top bipolar construct is accountability vs. irresponsibility, which received $19 \%$ of the vote. Despite the communal, liberating, non-institutional nature of Oxford House, no house is a loosely run, laissez-faire setting. It is a place of accountability. Through the rules and structure of the house, there is an internal, organizational structure is about mutual accountability for non-drug use or behavior that can put it at risk. Irresponsibility in this case is not about treatment settings, but life while using drugs and alcohol. The lower voted bipolar construct of structure vs. chaos provides a similar message.

The next two bipolar constructs, numbers 3 and 4, are interesting in the paradox they reveal. Surrender vs. self-will (\#3) received 16\% of the vote, and empowerment vs. powerlessness (\#4) 12\%. If we look at the foreground of the two sets of bipolar narratives, "surrender" seems almost the opposite of "empowerment" as does the contrast of "self-will" (mastery or power over one's actions) seem opposite of "powerlessness." Do the community constructs contradict one another? No, given the 12-step background of the community, surrendering to a high power and letting go of one's individual ego is in every way consistent with empowerment. Self-will may appear to scientists and professionals as the primary goal for individuals to achieve when overcoming addiction, but that is, in Rappaport's terms, the dominant narrative. The recovery

Table 1. Community Narrative Method Dichotomies listed in rank-order by vote.

\begin{tabular}{|c|c|c|}
\hline $\begin{array}{l}\text { OXFORD HOUSE } \\
\text { (RECOVERY } \\
\text { /ALTERNATIVE) }\end{array}$ & & $\begin{array}{l}\text { OPPOSITE } \\
\text { (DOMINANT) }\end{array}$ \\
\hline Growth & vs. & Stagnation \\
\hline Accountability & vs. & Irresponsibility \\
\hline Surrender & vs. & Self-Will \\
\hline Empowerment & vs. & Powerlessness \\
\hline $\begin{array}{l}\text { Behavior } \\
\text { Modification }\end{array}$ & vs. & Same Old \\
\hline Hope & vs. & Despair \\
\hline Self-Worth & vs. & Low Self-Esteem \\
\hline Sobriety & vs. & Using \\
\hline Support & vs. & No Support \\
\hline Family & vs. & Isolation \\
\hline Self-Sufficiency & vs. & Despair \\
\hline Secure Home & VS. & Homeless \\
\hline Democracy & vs. & Dictatorship \\
\hline Gratitude & vs. & Ungrateful \\
\hline Diversity & vs. & Uniform \\
\hline $\begin{array}{l}\text { Unconditional } \\
\text { Love }\end{array}$ & vs. & No Love \\
\hline Peer Pressure & vs. & Independence \\
\hline Structure & vs. & Chaos \\
\hline $\begin{array}{l}\text { narrative in this cas } \\
\text { egotistical self-cont } \\
\text { powerlessness. Sur }\end{array}$ & 1 & $\begin{array}{l}\text { elf-will, in the for } \\
\text { re equivalent to } \\
\text { "to a higher pow }\end{array}$ \\
\hline
\end{tabular}


empowerment are both high on the list of bipolar constructs underlying recovery narratives. Part of the interpretive process of using the Community Narration technique is comparing across the bipolar constructs and discussing such paradoxes with community members to get a richer understanding of the community's identity.

The interpretations of all the subsequent constructs are valuable. We just want to provide a sample here. Just one more is \#9, support vs. no support. At one point we as researchers believed social support was the primary beneficial impact of the Oxford House model and we hypothesized support as impacting \#8, sobriety vs. using. We still believe these relationships are vital to understand, but from using the participatory framework of the $\mathrm{CN}$ method, there is much else happening as well.

\section{Discussion}

The goal of Community Narration is to understand the identity or identities of an organization or community. The group's identity, signified by the bipolar constructs, is the lens through which the members see significant parts of their world, reflecting how the community narrates its shared story.

The results from the $\mathrm{CN}$ confirm the match between Kelly's (1955) personal construct theory and Rappaport's ideas behind community narratives. This synergy of personal construct and community narrative theories is most evident in the ways the underlying nature of bipolar constructs (opposing contrasts) to the dichotomy of alternative vs. dominant community narratives. Consistently with the Oxford House Community Narration, the left side of the bipolar construct represented the alternative narrative associated with the recovery setting. The other end represented the prior life, the dominant narrative, institutionalized treatment or a life of drug use, both of which have since been rejected by those who participated.

In the first instance, "growth" reflects the alternative narrative. The dominant narrative is reflected by "stagnation (or regression)." The right side, the dominant narrative construct adds an extra understanding of "growth" here. Growth was evidently a psychological moving forward, contrasted to being stuck or pulled back by life. This pattern fit across all bipolar constructs identified.

Several of the bipolar constructs referenced life in other treatment centers as the dominant community narrative pole. One was democracy vs. dictatorship. In two related instances, "despair" represented the dominant narrative, once opposed to the alternative narrative of hope and once to self-sufficiency. According to the participants, despair reflected their emotions and experiences as they revolved inside and out of various treatment centers.

Community Narration expands on the relationship between Kelly's and Rappaport's theories in other ways. Kelly believed all people are scientists living their lives testing hypotheses about the world in each new situation they encounter. People use these constructs, he believed, to reconstruct past experiences and interpret current interactions. Kelly also placed great emphasis on construals of the world through bipolar constructs as a way to anticipate future events. To the extent that Community Narration can help decipher how organizations and communities anticipate their next steps, practical uses present themselves. This includes working with a community to decide whether its mission is taking the group where it wants to go. Some organizations may be surprised about their own bipolar constructs that emerge, particularly if they reflect more dominant narratives, compared to the alternative narratives they might envisioned when the community first formed.

Kelly (1955) believed knowledge about bipolar construals could create more efficient organizational structures for the self; and the same is true of communities. Community Narration makes bipolar constructs more explicit to participants, helping them better articulate the psychological characteristics of their group, and may be used to help them better understand and build on the group's strengths.

Change does not come easily for all people and all groups. Kelly (1955) believed that individuals (and organizations/communities in this case) had greater or lesser abilities to change productively depending on their ability (or relatively inability) to incorporate new constructs into their cognitive system. Some psychological systems were, as Kelly named them, more or less "permeable", or flexible and open to adaptation. The permeability of a group's psychological structure may help a community become more open to new ideas or directions; or, in cases of greater impermeability, a more difficult time.

Community narrative constructs where the dominant (as opposed to the alternative) element is at the fore is likely in greater need of permeability. One may take the dichotomy found here of diversity $v$ s. uniform. Diversity reflects broad openness of a healthy system compared to an inflexible, institutional setting. Communal recovery, the case for Oxford House, compared to other traditional 
treatment facilities, allows for greater permeability in a less rigid and dogmatic system. This may reflect ways in which the Oxford House structure helps bring about growth, both as an organization and in the residents themselves.

Community Narration can also act as an aide to participatory action research. It helped us learn about how residents of a unique living model construed its benefits, more specifically, how we could better understand the process of growth through residents' greater social connectedness and tolerance for other ethnic and socioeconomic groups (Olson et al., 2009). Community Narration also helps us understand the group's perception of its own priorities. While the constructs are effective primarily as a focal-point for discussion, they can be returned to continually as organization progresses.

The possible variations on this method are endless. In summary, they help to identify a community's primary interests, and creative ideas toward improved group functioning. Community Narration can be used to uncover primary interests, detect problems and strengths, and determine subsequent steps toward action. Broadly defined, Community Narration is about understanding the "culture" of a setting or group, that is, its belief systems, values, social norms, and practices (Maton, 2000; Sarason, 1971).

Community Narration is a starting point for exploring a group's personal and community values, andthrough identity, cognition, and the empowering nature of narratives - to help bring about change.

\section{References}

Fetterman, D. M., Kafterian, S. J., \& Wandersman, A. (1996). Empowerment evaluation: Knowledge and tools for self-assessment and accountability.

Thousand Oaks, CA: Sage.

Fetterman, D.M. \& Wandersman, A. (Eds.) (2005). Empowerment evaluation: Principles and practice. N.Y.: Guilford.

Harré, N, Bullen, P., \& Olson, B. (2006).

Storytelling: A workshop for inspiring group action. In R. McNair (Ed.) Working for Peace: A Handbook of practical psychology and other tools. Atascadero, CA: Impact Publishers.
Humphreys, K. (2000). Community narratives and personal stories in Alcoholics Anonymous. Journal of Community Psychology, 28, 495-506.

Jason, L. A., Davis, M. I., Ferrari, J. R., Bishop, P. D. (2001). Oxford House: A review of research and implications for substance abuse recovery and community research. Journal of Drug Education, 31(1), 1-27.

Kelly, G. A. (1955). The Psychology of personal constructs (2 vols.). New York: W.W. Norton.

Kalekin-Fishman, D. \& Walker, B. (Eds.) (1996). The construction of group realities: Culture, society, and personal construct theory (pp. 309-322).

Melbourne, FL: Krieger.

Maher, B. (1969). (Ed.). Clinical psychology and personality: The selected papers of George Kelly. New York: John Wiley.

Maton, K. I. (2000). Making a difference: The social ecology of social transformation. American Journal of Community Psychology, 28, 25-57.

McAdams, D. P. (2009). The person: An introduction to the science of personality psychology (5th Ed.). Wiley.

McAdams, D. P., \& Olson, B. D. (2009). Personality development: Continuity and change over the life course. Annual Review of Psychology, 61, 5.1-5.26.

Olson, B. D., Jason, L. A., Davidson, M., \& Ferrari, J. R. (2009). Increases in tolerance within naturalistic, intentional communities: A randomized, longitudinal examination. American Journal of Community Psychology, 44, 188-195.

Olson, B. D., Jason, L. A., Ferrari, J. R., \& Hutcheson, T. D. (2005). Bridging professional and mutual-help: An application of the transtheoretical model to the mutual-help organization. Applied and Preventive Psychology, 11, 167-178.

Patton, M.Q. (2002). Qualitative research and evaluation methods. CA: Sage.

Rappaport, J. (2000). Community narratives: Tales of terror and joy. American Journal of Community Psychology, 28(1), 1-24.

Sarason, S. B. (1971). The culture of the school and the problem of change. Boston: Allyn \& Bacon. 